\section{PTU-224 ERRORS IN ENDOSCOPY, SCOPE TO IMPROVE? AN ANALYSIS OF NON-TECHNICAL SKILLS AND SAFETY CHECKS IN ENDOSCOPY}

doi:10.1136/gutjnl-2012-302514c.224

${ }^{1,2} \mathrm{M}$ K Matharoo, ${ }^{*}{ }^{1} \mathrm{~A}$ Haycock, ${ }^{3} \mathrm{~N}$ Sevdalis, ${ }^{1} \mathrm{~S}$ Thomas-Gibson. ${ }^{1}$ Endoscopy, St. Mark's Hospital, Harrow, UK; ${ }^{2}$ Centre for Patient Safety and Service Quality (CPSSO), Imperial College, London, UK; ${ }^{3}$ CPSSQ, Imperial College, London, UK

Introduction Existing evidence shows that many medical errors are avoidable and a systematic approach using safety checks such as the WHO Surgical Safety Checklist can reduce adverse events. ${ }^{1}$ Research within high-risk industries has illustrated that errors with significant impact on safety often relate to non-technical skills (NTS) rather than technical ability. By focusing on the key NTS in Endoscopy, we hypothesise that team function ${ }^{2}$ will be enhanced, patient safety improved and errors reduced.

Methods Current safety practices in Endoscopy were evaluated prospectively via (1) Assessment of current safety checks and (2) Analysis of safety enhancing NTS (ie, behaviours), based on a pilot study. Behaviours deemed to be "safety checks" (SC) that impact positively on patient care were determined by expert consensus. Endoscopists were observed and their checking behaviours assessed by two independent clinical observers: (1-4 scale, $4=$ "gold standard" SC of "cross checking" with a colleague, $1=$ no discernible attempt to perform an SC). Endoscopists NTS were assessed quantitatively (1-4 scale) using a validated framework. ${ }^{3}$ In addition any errors, near misses or adverse events (AE) were qualitatively recorded for each procedure.

Results 22 lists were observed and 90 procedures analysed from a representative sample of 16 Endoscopists. In total 1218 opportunities to perform a safety check were identified. The "gold standard check" was only performed in 9\% of instances. In 37\% of episodes no check was completed. Endoscopists and nurses performed similar checks separately, often without communication. ENTS scores varied, (mode $=3, \min =1 \max =4)$. Endoscopists scoring higher on NTS were more likely to perform safety checks (correlation coefficient $r=0.82 \mathrm{p} \leq 0.001$ ). 41 safety incidents were observed and $27 \%$ occurred in the lists where the Endoscopist scored an NTS of 1 or 2 and $0 \%$ occurred in those with an NTS of 4.

Conclusion This study demonstrates wide variability in safety checks and non-technical skills in Endoscopy. There appears to be a relationship between robust safety checks and good NTS. Further research should focus on the relationship between technical (DOPS) and non-technical (ENTS) skills and whether training in NTS for Endoscopists can reduce adverse events and improve their safety behaviour.

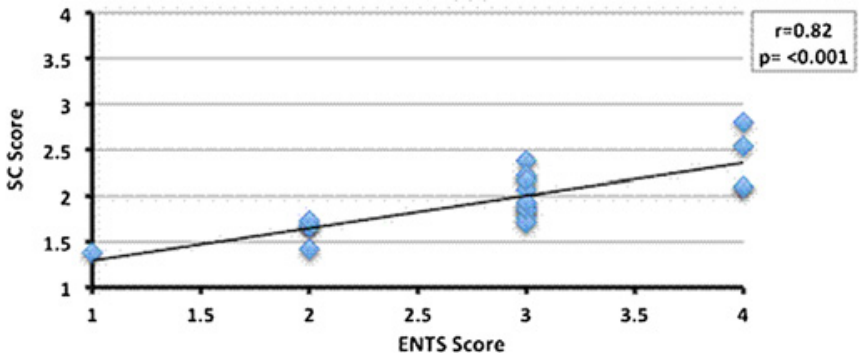

Abstract PTU-224 Figure 1 Endoscopic non-technical skills (ENTS) vs safety check (SC) scores.

Competing interests M Matharoo Grant/Research Support from: This group has received funding from the NHS Bowel Cancer Screening Research Programme for development of non-technical skills awareness $\&$ team training, Conflict with: The Freemasons Grand Charity, A Haycock: None declared, N Sevdalis: None declared, S Thomas-Gibson: None declared.

\section{REFERENCES}

1. Haynes, et al. A Surgical Safety Checklist to Reduce Morbidity \& Mortality in a Global Population. NEJM 2009:360

2. Haycock, et al. Effective teamworking in gastroenterology. Frontline Gastroenterology 2012.

3. Haycock. Enhancing Professional behaviour in gastrointestinal endoscopy: development of a behavioural marker tool for assessment of ENTS. Gut 2010;59

\section{PTU-225 REFERRAL TO GI ENDOSCOPY FOR ANAEMIA, DOES THE MCV REALLY MATTER?}

doi:10.1136/gutjnl-2012-302514c.225

${ }^{1,2} \mathrm{M}$ Walshe, ${ }^{* 1,2} \mathrm{~J}$ Nolan, ${ }^{1,2} \mathrm{C}$ Lahiff, ${ }^{1,2} \mathrm{P}$ MacMathuna. ${ }^{1} \mathrm{G} /$ Unit, Mater Misericordiae University Hospital, Dublin, Ireland, ${ }^{2}$ University College Dublin, Dublin, Ireland

Introduction Anaemia is a common indication for referral to GI endoscopy services. Appropriate triage of these referrals may be aided by further characterisation of the anaemia and review of iron studies. However, value of the MCV in predicting a positive endoscopic finding has been questioned. Our aim was to determine predictors of positive endoscopic findings in patients referred to a single centre for investigation of anaemia in a 6-month period, in relation to full blood count (FBC), iron studies, and patient age.

Methods A retrospective cohort study examining endoscopic procedures performed where anaemia without an obvious cause was the primary indication. Patients with overt GI haemorrhage were excluded. Data were extracted from an electronic database and specific parameters included demographic variables, indication(s) for endoscopy, haematological values, iron studies, and endoscopic findings. Positive endoscopy was defined as a finding considered responsible for the anaemia. Analysis was performed using GraphPad Prism and Microsoft Excel.

Results A total of 359 endoscopic procedures (210 gastroscopies, 137 full colonoscopies, 12 left colonoscopies) were performed in 243 patients. In 48/243 (20\%) patients, a cause for anaemia was found 10/243 (4\%) had malignancy; [3/243 (1\%) gastric ca, 7/243 (3\%) colorectal ca]. 38/243 (16\%) had a non-malignant cause of anaemia, including gastric ulcer $15 / 243(6 \%)$, angiodysplasia $13 / 243(5 \%)$, duodenal ulcer 4/243 (2\%), and coeliac disease 2/243 (1\%). Endoscopy was normal or revealed incidental findings in the remaining 195/243 (80\%) patients. Older age and higher RDW were significantly associated with positive endoscopy ( $p=0.006, p=0009$ respectively). While the association with lower serum $\mathrm{Hb}$ trended towards significance $(p=0.07)$, no association between $M C V$ and positive endoscopy was observed $(p=0.87)$. Low serum ferritin and MCV were significantly associated with malignancy $(p=0.05$, $\mathrm{p}=0.05)$, as was a higher RDW ( $\mathrm{p}=0.03)$.

Conclusion While a low MCV was significantly associated with malignancy on GI endoscopy, it was not significantly associated with a positive endoscopy overall. RDW is a good predictor of positive endoscopy with regard to both malignant and non-malignant causes of anaemia. Hence, the performance of GI endoscopy can be considered in the context of a normal MCV.

Competing interests None declared.

PTU-226

COLORECTAL CANCER SCREENING IN A MODERATE RISK POPULATION; WHAT IS THE POLYP YIELD AND WHAT IS THE OPTIMUM SCREENING INTERVAL?

doi:10.1136/gutjnl-2012-302514c.226

${ }^{1,2} \mathrm{M}$ Walshe, ${ }^{* 1,2} \mathrm{R}$ Moran, ${ }^{1,2} \mathrm{~V}$ Swan, ${ }^{1,2} \mathrm{~J}$ Trickovic, ${ }^{1,2} \mathrm{~B}$ Kelleher, ${ }^{1,2} \mathrm{P}$ MacMathuna ${ }^{1}$ GI Unit, Mater Misericordiae University Hospital, Dublin, Ireland; ${ }^{2}$ University College Dublin, Dublin, Ireland

Introduction Colorectal cancer (CRC) screening has been shown to reduce mortality from CRC. Many people with a family history of 